Research Article

\title{
Ion Cyclotron Resonance: Results and Prospects for Psychiatry
}

\author{
Mario Betti, MD', Marco Paolo Carlo Picchi, PhD², Marco Saettoni, MD', and Alessandro Greco, MD ${ }^{*}$ \\ ${ }^{1}$ Responsible UFSMA, Adults Mental Health Functional Unit, Valle del Serchio, USL Nord Ovest, Italy \\ ${ }^{2}$ Psychologist, Member ECONA, Inter-University Research Institution on the Cognitive Processing in Artificial and Natural \\ Systems, Italy
}

${ }^{3}$ Psychiatrist Medical Director UFSMA, Valle del Serchio, USL Nord Ovest, Italy

${ }^{4}$ Medical Director A.P.S.P. Cles Trento, Italy

\begin{abstract}
Background: The Ion Cyclotron Resonance-like (ICR-like) phenomenon is a physical model for the ionic currents through cellular membranes obtained by application of very weak, extremely low frequency electromagnetic fields (ELF-EMF). It was proposed in 1984 by the American physicist Abraham Liboff as a hypothesis to explain the results of the physicists Adey and Blackman in their studies of these currents. Liboff postulated that the ELF-EMF applied in the experiments interacted with the geomagnetic field (GMF), inducing facilitated movement of ion species through cellular membranes and so acting as modulators for the degree of movement of the ions.

As is well known, there is still much to learn about how ELF-EMF interact with living structures. In the current state-ofart the ICR-like phenomenon represents a very valid explanation of this interaction and deserves more in-depth studies into its application in medical fields. Among these psychiatry certainly offers numerous possibilities for the application of ELF-EMF.

In recent decades psychiatric practice has undergone a revolution, shifting its operative focus from the traditional psychiatric hospitals to services dispersed within the community. New medical, social, rehabilitative, and psychotherapeutic treatment methods have been tested. Among these the use of ELF-EMF has been found to offer both great versatility and interesting potential for therapeutic use.

An emblematic example is the so-called "repetitive transcranial magnetic stimulation" (rTMS). It was introduced by Anthony Barker (University of Sheffield, UK) in 1985 [1] as a non-invasive instrument to activate the motor cortex, measure its connectivity, excitability, and assess the integrity of the motor pathways. Ever since, its use in neurology, clinical neurophysiology, and psychiatry has been spreading into both research and more strictly clinical applications [2]. In the psychiatric field it has been successfully applied for various disorders, including for example:
\end{abstract}

- Treatment-resistant depression

- Depression associated with metabolic disorders

- Depression in which drugs have to be avoided (hepatic or renal insufficiency, etc.)

- Seasonal affective disorder

- Psychosis

- Negative symptoms

- Auditory hallucinations

- Obsessive-compulsive disorder

This and other examples of the application of ELF-EMF led the present authors to hypothesize that ICR-like might not only be a valid approach to psychiatric diseases, but also a good starting point to describe how ELF-EMF generate positive effects in these pathologies, and so cast new light on the physiology and psychopathology of the mind.

This article presents the results of a naturalistic observational study to assess the effectiveness of this methodology in the treatment of psychopathological disorders.

Aim: The main aim was to confirm the positive results obtained in a previous study, increasing the size of the sample set and observing the results for a restricted group of subjects with more homogenous symptoms and ambient conditions of treatment.

Copyright: (c) 2019 Betti M, et al. This is an open-access article distributed under the terms of the Creative Commons Attribution License, which permits unrestricted use, distribution, and reproduction in any medium, provided the original author and source are credited. 


\begin{abstract}
A further aim was to discriminate response to the treatment on the basis of severity of symptoms, and thus its respective impact on the functional and organic psychopathogenic components.
\end{abstract}

Materials and methods: A sample set of 46 patients of the Adult Functional Mental Health Unit of Valle del Serchio (ASL 2 Lucca, Italy) were studied. They were treated with an experimental therapy using low intensity, extremely low frequency electromagnetic fields (ELF-EMF). The results were assessed on the basis of the Brief Psychiatric Rating Scale (BPRS) conducted before, during and after treatment.

Results: The results revealed a significant improvement in the clinical picture, achieved in particular during the initial period of treatment. Treatment appears to be more effective on patients characterized by a very serious clinical presentation.

Conclusions: The results obtained encourage continuation of studies into the therapeutic outcomes of the applied methodology, further extending the number of samples and including control groups. The therapeutic results appear more marked in the more serious clinical pictures, in which the organic component has greater bearing than the functional component.

\section{Introduction}

\section{The therapeutic use of extremely weak magnetic fields and the ion resonance effect}

In recent decades psychiatric practice has undergone a revolution, shifting its operative focus from the traditional psychiatric hospitals to services dispersed within the community. New medical, social, rehabilitative, and psychotherapeutic treatment methods have been tested. However, one area that has been scarcely explored is the application of biophysical therapies, and in particular treatments based on pulsed low intensity, extremely low frequency electromagnetic fields (ELF-EMF). This despite the fact that for some time there have been good theoretical and pathogenetic grounds for this, as well as clinical observations that support the effectiveness of this methodology [3].

Studies conducted by Liboff in the USA starting from the 1980 s demonstrated that ELF-EMF in combination with the geomagnetic field are capable of generating an effect defined as ion resonance-like (for simplicity referred to below as ion resonance), activating the transfer of ions through cell membranes and facilitating the process of repolarization [4]. The ion resonance effect involves the interaction of a static magnetic field (geomagnetism) with a series of pulsed or dynamic waves produced artificially. It is thus referred to as "combined magnetic fields" (CMF). This phenomenon represents the "Rationale" on which the scientific research and clinical studies have been founded over the last thirty years [5].

It is important to note that the ion resonance effect is only generated within a given range of intensities, frequencies, and waveforms, which vary with the variation of the geomagnetic field at any given spatial location.

By measuring the electrical impedance of an organism followed by computer processing, it is possible to select and record a sequence of electromagnetic waves on a data card, suitable for generating the ion resonance effect for any individual person in a given location. This type of treatment is therefore highly focused and customized.

\section{The experimental context}

The study reported in this article took place at the Azienda
Sanitaria Locale (ASL) [Local Health Authority] n. 2 of Lucca (Italy), at the Adult Functional Mental Health Unit of Valle del Serchio, in a Public Service that operates prevalently in the local community outside the hospital context. Starting from the end of the 1990s, with the support of Tuscany Region, the above mentioned service initiated an experimental procedure that made it possible to combine traditional treatments, essentially psychopharmacological, with other therapeuticrehabilitative approaches, including cognitive-behavioral, systemic-relational, psycho-corporeal, and transpersonal psychotherapies, mindfulness, art-therapies, and psychoeducational interventions.

In 2009 biophysical treatments were also introduced and in particular light therapy and ion cyclotron resonance-like (ICR-like) treatments. While for the former there had been a substantial scientific literature for some decades [6-9], ICRlike had only been introduced into clinical practice a few years previously and was still in the research and experimental stage. In particular, this methodology had not yet been systematically applied in the psychiatric sector. However, the following series of factors encouraged the authors to introduce the technique and initiate experimentation.

a) ICR-like is employed successfully in various medical fields, in particular in oncology, orthopedics, and neurology: Experimental data demonstrates its effectiveness and near absence of side effects [10]. Of particular interest for the present research were its effects on autism [11], Parkinson's disease [12], Alzheimer's dementia [13], and multiple sclerosis $[14,15]$.

b) The use of extremely weak magnetic fields (of intensities and frequencies similar to those of the geomagnetic field) are perfectly compatible with the absorption capacity of

*Corresponding author: Alessandro Greco, MD, Medical Director A.P.S.P. Pellizzano, APSP Santa Maria Cles, Via E. Chini 37, Cles (TN) CAP 38023, Italy

Accepted: March 26, 2019

Published online: March 28, 2019

Citation: Betti M, Picchi MPC, Saettoni M, et al. (2019) Ion Cyclotron Resonance: Results and Prospects for Psychiatry. J Psychiatry Treat Res 1(1):16-24 
the human organism.

c) The technique is acknowledged as effective by the "Food and Drug Administration".

d) Its mechanisms of action provided a rational basis for hypothesizing its effectiveness in psychiatric disorders.

e) Its ease of application, practicality and non-invasive nature encouraged its use.

\section{Hypotheses on the mechanisms of action and rational bases of ICR-like effects}

On the strength of numerous studies conducted in recent years, at least four mechanisms of action can be hypothesized, offering the same number of rational foundations to justify the introduction of this methodology into the field of psychiatric treatment [16].

Action on membrane potential and on enzymatic cellular processes: On the basis of current biophysical knowledge [17] it is known that pulsing low intensity and low frequency electromagnetic fields act by modifying the membrane potential of nerve cells.

There is stimulation of the sodium-potassium pump and other enzymatic systems in the cellular membrane and in the intracellular organelles, in particular in the microtubules, with modification of permeability and promotion of ionic exchange. It is likely that these changes are relevant for the re-activation of dysfunctional neural circuits.

A mechanism of action of this type was hypothesized to explain the disinhibiting effects of electroshock therapy [18]. According to this theory the electrical current acts on the electromagnetic fields of the fibers and nerve cells, modifying the flow of ions through the neuron membranes. It is also assumed that, together with its harmful effects, electroshock has a protective effect on nerve cells, increasing the secretion of neurotrophic factors into the limbic system [19].

ICR-like also acts by modifying the electromagnetic fields of cellular membranes, but unlike electroshock it makes it possible to provide finely modulated treatments, avoiding invasive and potentially harmful interventions, like those produced by massive doses of chemical substances or electrical currents. Furthermore, thanks to the assessment of the impedance of the tissues of the organism and the selection of waves capable of generating the ion cyclotron effect, it is possible to target treatments for individuals and for the specific stage of their disorder.

It should also be noted that ICR-like does not act exclusively on certain structures but instead its action is widespread over all cellular membranes, from endothelial cells to fibroblasts, to nerve cells, and glial cells.

Consequently, diverse non-specific effects can be expected on different structures and pathologies. The action on microtubules is particularly interesting, since it encourages the expression of biochemical messages between nucleic acids, ribosomes, and membrane structures [20].

This mechanism can explain therapeutic effects at an ear- ly stage, and a clinical improvement can already be hypothesized after the first treatment sessions.

Action on chemical mediators: Studies conducted with deep, low frequency, repetitive transcranial magnetic stimulation (rTMS), demonstrate that this methodology induces an increase in chemical mediators like dopamine and melatonin $[21,22]$. On the basis of a re-assessment of 15 meta-analyses (PubMed 2001-2010), it can be established that rTMS appears to be an effective cerebral stimulation technique for the treatment of major depressive disorder [23]. It is feasible that an analogous action is also exercised by ICR-like, which offers the additional advantage of selecting customized waves according to the impedance of each individual organism.

Stimulation of neurotrophic factors: Another effect linked to electromagnetic fields involves the stimulation of neurotrophic factors, which could explain a more gradual effect extended through time. The studies initiated by Liboff $[4,24,25]$ clearly indicate that electricity, available both inside the organism and produced by processes of internal transduction, is used in the physiological processes of living systems to stimulate the phenomena of growth, repair, and regeneration.

It was observed that electromagnetic fields stimulate the production of neurotrophic factors, capable of increasing cellular growth and the regeneration of diverse tissues, in particular neuronal and glial.

This ensures protection against atrophy, induced by depression and psychotic disorders, evolving towards dementia. In the past this type of action was also attributed to electroshock, as an explanation for the secretion of neurotrophic factors observed in certain cases [19].

According to the theoretical suppositions it is possible to hypothesize an early clinical response associated with membrane polarization effects, and a later effect associated with neurotrophic induction. The trophic action on neuronal and glial tissues could explain a more delayed effect extended over time, with the possibility of improvements after months or even years.

Reduction of oxidative stress: Of particular importance is a protective action in relation to oxidative stress. Lipids, proteins, carbohydrates, and nucleic acids undergo oxidation, generating metabolic products that can damage cells. While on one hand oxygen is essential for the human metabolism, on the other hand it can become harmful due to the numerous uncontrolled oxidation reactions (Autoxidation) in the presence of the so-called reactive oxygen species (ROS) that cause cellular stress. Alongside numerous internal diseases, oxidative stress also encourages the onset and aggravation of psychiatric disorders, including schizophrenia and degenerative dementia [26].

Ion cyclotron resonance-like treatment induces an improvement in the oxidation balance with an increase in antioxidant factors and a reduction in pro-oxidant factors.

As a consequence there is also a reduction in the levels of 
free radicals in the blood. This mechanism is another reason to foresee medium- to long-term therapeutic effects.

\section{Aims of the Study}

On the basis of the mechanisms of action of ICR-like and the clinical results obtained previously, it was reasonable to expect significant therapeutic effects in both the shortand medium-term. The ease of use of the instrument, the sensations of relaxation and wellbeing reported by numerous users at the end of each session, and high human tolerance of extremely low electromagnetic fields offered reassurance when proposing the application of this methodology to a series of patients who had proven resistant to pharmacological treatment. Furthermore, taking into account the electrodynamics action on cellular tissues and in particular on nervous fiber, it was expected to achieve obvious effects especially in the more serious cases, in which the biophysical component was more seriously compromised.

\section{Materials and Methods}

\section{Introduction}

An initial observational study was started as early as 2009, involving 33 patients and presented in 2010 at the Third National Convention of the Italian Society for Biophysical Electrodynamics. The results demonstrated the statistically significant effectiveness of this treatment, encouraging the team to continue their research [27].

This initial study examined a sample of 33 patients, affected by chronic psychiatric disorders, mainly psychotic in nature, and resistant to pharmacological treatment, all patients of the Mental Health Centre for at least 5 years. Each treatment program included 20 sessions of variable duration from 18 to 54 minutes.

During both the preliminary observational study and the present one, a SEQEX ${ }^{\circledR}$ device was used, produced and distributed by the Italian company S.I.S.T.E.M.I. srl (Trento, Italy), certified CSQ ISO-13485.This device has the capacity to produce complex electromagnetic fields by an analogical mechanism across a frequency range of 1 to $80 \mathrm{~Hz}$ and at intensities of 1 to $20 \mu \mathrm{T}$. The field parameters are tested by the manufacturing company using specialized instrumentation: the device used for this purpose is a Gauss meter GM 08 produced by the company Hirst. The administration of the electromagnetic field produced by the control unit of the treatment device (on which the parameters of the electromagnetic field can be set), is achieved through a mat inside of which a Helmholtz coil generates the ELF-EMF. The patient lies down on the mat and receives non-focused total body treatment with the desired electromagnetic fields.

In both the preliminary observational study and the present one, the same complex treatment was applied comprising 9 intensity and frequency pairs (called "steps"), with a time-on for presence and time-off for absence of static field. Combinations of the following waveforms were used in the different steps: sinusoidal, sawtooth, square. The characteristics are set out in the Table 1.
While there exists the possibility of administering patients both total body treatment (using a mat) combined with local stimulation (using various accessories that administer the same electromagnetic field as the mat), all the subjects involved were only treated with non-focused total body therapy using a mat.

The therapeutic protocol was 3 treatments per week for a total of 20 treatment sessions per patient. Assessments were made using the BPRS (Brief Psychiatric Rating Scale), at TO T1 T2, respectively the start, after the tenth session, and on termination. The results obtained during the study suggest a positive effect of treatment, expressed in particular during the first half of the treatment program. No differences in results were observed in relation to the length of treatment sessions, and so it was considered preferable to opt for times between 27 and 36 minutes.

\section{Study design}

This article presents a second study, aiming to probe the results of the previous observations in greater depth and to conduct other assessments. This was an explorative study conducted in a real context, using quantitative criteria according to the so-called "naturalistic" or "quasi-scientific" observational method, in which all the variables are not manipulated or subject to controls, but instead the changes in a group of subjects undergoing treatment are observed and assessed. The collection of data and assessment of outcomes were conducted within a short time.

\section{Setting}

A total of 104 treatment programs were initiated over the period from Aug $6^{\text {th }}, 2009$ to Dec $22^{\text {nd }} 2010$ in a residential psychiatric center managed by the Mental Health Service. Of these, 43 treatments were on patients temporarily resident in the center, while the remaining 61 were outpatients. Some patients were treated more than once.

Patients that underwent ICR-like treatment were all under the care of the Mental Health Service for at least two years and had exhibited resistance to pharmacological treatments, with a tendency towards chronicity and periodic exacerbation of symptoms.

Table 1: Table of electromagnetic fields used for treatment. Each step had the same duration (2-3-4-5 minutes). Time-on and -off are measured in seconds.

\begin{tabular}{|l|l|l|l|l|}
\hline Step & Intensity $(\boldsymbol{\mu} \mathbf{T})$ & Frequency $(\mathbf{H z})$ & Time-on & Time-off \\
\hline 1 & 15 & 50 & 4 & 3 \\
\hline 2 & 15 & 10 & 4 & 3 \\
\hline 3 & 12 & 50 & 4 & 3 \\
\hline 4 & 12 & 20 & 4 & 3 \\
\hline 5 & 12 & 40 & 4 & 3 \\
\hline 6 & 10 & 40 & 4 & 3 \\
\hline 7 & 10 & 30 & 4 & 3 \\
\hline 8 & 10 & 20 & 4 & 3 \\
\hline 9 & 10 & 10 & 4 & 3 \\
\hline
\end{tabular}


Citation: Betti M, Picchi MPC, Saettoni M, et al. (2019) Ion Cyclotron Resonance: Results and Prospects for Psychiatry. J Psychiatry Treat Res $1(1): 16-24$

Table 2: Number of treatments divided for protocol duration.

\begin{tabular}{|c|c|c|c|c|c|}
\hline \multicolumn{2}{|c|}{ Duration of treatment (individual session) } & \multirow{2}{*}{\begin{tabular}{|l|} 
Frequency \\
1 \\
\end{tabular}} & \multirow{2}{*}{$\begin{array}{l}\text { Percent } \\
2.2\end{array}$} & \multirow{2}{*}{\begin{tabular}{|l|} 
Valid percent \\
2.2 \\
\end{tabular}} & \multirow{2}{*}{$\begin{array}{l}\text { Cumulative percentage } \\
2.2\end{array}$} \\
\hline Valid & Minutes 18 & & & & \\
\hline & Minutes 27 & 34 & 73.9 & 73.9 & 76.1 \\
\hline & Minutes 36 & 4 & 8.7 & 8.7 & 84.8 \\
\hline & Minutes 45 & 6 & 13.0 & 13.0 & 97.8 \\
\hline & Minutes 54 & 1 & 2.2 & 2.2 & 100.0 \\
\hline & Totals & 46 & 100.0 & 100.0 & \\
\hline
\end{tabular}

Table 3: Patient enrolled divided for diagnoses (based on ICD9, group 1).

\begin{tabular}{|c|c|c|c|}
\hline \multicolumn{2}{|c|}{ Diagnoses ICD 9 (group 1) } & \multirow{2}{*}{\begin{tabular}{|l} 
Frequency \\
0
\end{tabular}} & \multirow{2}{*}{\begin{tabular}{|l|} 
Percentages \\
2.2 \\
\end{tabular}} \\
\hline Valid & Senile and pre-senile organic psychotic states & & \\
\hline & Other psychotic states (chronic) & 0 & 2.2 \\
\hline & Schizophrenic psychoses & 10 & 13.0 \\
\hline & Affective psychoses & 15 & 32.6 \\
\hline & Psychoses with specific origins in infancy & 0 & 4.3 \\
\hline & Neurotic disorders & 9 & 19.6 \\
\hline & Personality disorders & 6 & 13.0 \\
\hline & Adjustment disorders & 6 & 13.0 \\
\hline & Totals & 46 & 100.0 \\
\hline
\end{tabular}

There had been no changes to the current pharmacological therapy within the two months prior to the start of treatment, and no significant variations in symptoms had been recorded. All the participants were suitably informed and consented to treatment.

\section{Treatment}

Each treatment program included 20 sessions of variable duration, but mostly of 27 minutes (Table 2). The therapeutic protocol applied in the present second study is described at the start of this chapter. The intensities, frequencies, timeson, and times-off of the electromagnetic fields were not changed. The subjects involved were treated 3 times per week for a total of 20 treatment sessions with non-focused total body treatment. All treatment sessions were administered using the same device in the same room.

The variable duration of the treatment sessions was dictated by the compliance of the subjects.

Assessments were made using the BPRS (Brief Psychiatric Rating Scale), with TO T1 T2 respectively at the start, after ten sessions, and on termination.

\section{Assessment criteria}

The result assessments were conducted using the Brief Psychiatric Rating Scale version 4.0, in its Italian adaptation, care of Morosini [28]. The instrument consists of a semistructured interview and is accompanied by a manual that provides precise indications for accurate numerical quantification of the level of seriousness of symptoms.

The reference time period for the assessment was the week prior to the same. It was compiled by psychiatric doctors
Table 4: Age of patients enrolled (group 1).

\begin{tabular}{|l|l|l|l|l|l|}
\hline & N & Minimum & Maximum & Average & Std. Deviation \\
\hline Age & 39 & 22.00 & 74.00 & 50.9231 & 14.54102 \\
\hline
\end{tabular}

Table 5: Patient enrolled divided for gender (group 1).

\begin{tabular}{|c|l|l|l|}
\hline \multicolumn{2}{|c|}{ Gender (group 1) } & Frequency & Percentages \\
\hline \multirow{3}{*}{ Valid } & Male & 15 & 32.6 \\
\hline & Female & 31 & 67.4 \\
\cline { 2 - 4 } & Totals & 46 & 100.0 \\
\hline
\end{tabular}

who took into consideration (as indicated in the manual) not only the patient's answers but also all the information in their possession, including contact with family members and clinical records. There are 24 items comprising the assessment scale, with scores from 1 to 7 . The test produces a score that ranges from 24 to 148 . In line with literature [29,30] a reduction in score equal to or above $44 \%$ was considered a "marked improvement", a reduction between $24 \%$ and $44 \%$ was considered a "mild improvement", a change in score of $+/-24 \%$ was considered to be an index of a "stable" condition, and an increase in score equal to or above $24 \%$ was considered an index of "deterioration". For the calculation of the evolution percentages, 24 points were subtracted from the overall score of each assessment.

Group 1: $\mathrm{N}=46$ patients characterized by various types of diagnoses (Table 3 ) with a prevalence of affective psychosis ( $\mathrm{n}$ $=15$ ) and neurotic disorders ( $n=9), 15$ male and 31 female, with an average age of 50.9 years (Table 4 and Table 5). These patients underwent at least one ICR-like treatment cycle and follow up assessment. 
Citation: Betti M, Picchi MPC, Saettoni M, et al. (2019) Ion Cyclotron Resonance: Results and Prospects for Psychiatry. J Psychiatry Treat Res $1(1): 16-24$

Table 6: Patient enrolled divided for diagnoses (based on ICD9, group 1).

\begin{tabular}{|l|l|l|l|}
\hline Diagnoses ICD 9 (group 2) & Frequency & Percentages \\
\hline Valid & Schizophrenic psychoses & 1 & 8.3 \\
\hline & Affective psychoses & 5 & 41.7 \\
\cline { 2 - 4 } & Psychoses with specific origins in infancy & 1 & 8.3 \\
\hline & Personality disorders & 1 & 8.3 \\
\hline & Adjustment disorders & 4 & 33.3 \\
\hline & Totals & $\mathbf{1 2}$ & $\mathbf{1 0 0 . 0}$ \\
\hline
\end{tabular}

Table 7: Age of patients enrolled (group 2).

\begin{tabular}{|l|l|l|l|l|l|}
\hline & N & Minimum & Maximum & Average & Std. deviation \\
\hline Age & 10 & 25.00 & 71.00 & 45.9000 & 16.75609 \\
\hline
\end{tabular}

Table 8: Patient enrolled divided for gender (group 2).

\begin{tabular}{|l|l|l|l|}
\hline Gender (group 2) & & Frequency & Percentages \\
\hline Valid & Male & 5 & 41.7 \\
\cline { 2 - 4 } & Female & 7 & 58.3 \\
\cline { 2 - 4 } & Totals & $\mathbf{1 2}$ & $\mathbf{1 0 0 . 0}$ \\
\hline
\end{tabular}

Table 9: BPRS score of group 1.

\begin{tabular}{|l|l|l|l|l|l|}
\hline \multicolumn{2}{|l|}{ Statistics for paired samples (group 1) } & Average & N & Difference & Significance (2-tailed) \\
\hline Pair 1 & Overall BPRS score first cycle (t0) & 49.6304 & 46 & & \\
\cline { 2 - 5 } & Overall BPRS score second cycle (t1) & 41.4565 & 46 & 8.18 & $\leqq 0.01$ \\
\hline
\end{tabular}

Table 10: Skills improved on BPRS score after treatments.

\begin{tabular}{|c|c|c|}
\hline Clinical evolution & Range & t0-t1 \\
\hline Deterioration & Score increased by at least $24 \%$ & $15.2 \%$ \\
\hline Stable & Score within $+/-24 \%$ of the initial & $26.1 \%$ \\
\hline Slight improvement & Score diminished by 24 to $44 \%$ & $19.6 \%$ \\
\hline Marked improvement & Score diminished by at least $44 \%$ & $39.1 \%$ \\
\hline
\end{tabular}

Group 2: N = 12 patients affected by psychotic disorders (affective or schizophrenic, Table 6) underwent two cycles of ICR-like treatment with relative follow up assessments. They did not have any changes in pharmacological and/or rehabilitative-behavioral therapy during the period in question and they were not resident at the psychiatric facilities during treatment. The sample included 5 males and 7 females, with an average age of 45.9 years (Table 7 and Table 8 ).

\section{Statistical analysis instruments}

The entry of data, screening, and processing of output were conducted by a psychologist expert in statistical analysis using the PASW (Predictive Analysis SoftWare) package, equivalent to version 18.1 of the SPSS (Statistical Package for Social Sciences).

Assessments were conducted before starting the first treatment cycle (t0), after the first cycle (t1), and after the second cycle (t2).

The comparison between the sample averages at t0 and $\mathrm{t} 1$ and between the sample averages at $\mathrm{t} 1$ and $\mathrm{t} 2$ was conducted using Wilcoxon's Test applied to paired samples with a two-tailed significance test.

The correlation index $r$ with assessment of significance on two tails was used to test the correlation between the initial score and the evolution of scores after the first therapy cycles (scores at t0 correlated with t1-t0) exclusively for group 1.

\section{Results}

In group 1 (patients with various pathologies and without controls for incidental variations in pharmacological and/ or rehabilitative-behavioral therapy) the average scores obtained using the BPRS were 49.63 at to and 41.45 at t1 (difference $=8.18 \mathrm{p} \leqq 0.01$ ) (Table 9).

The clinical significance in the evolution of the scores indicated a deterioration in $15.2 \%$ of cases, a substantial stability in $26.1 \%$, a slight improvement in $19.6 \%$, and a marked improvement in 39.1\% (Table 10). 


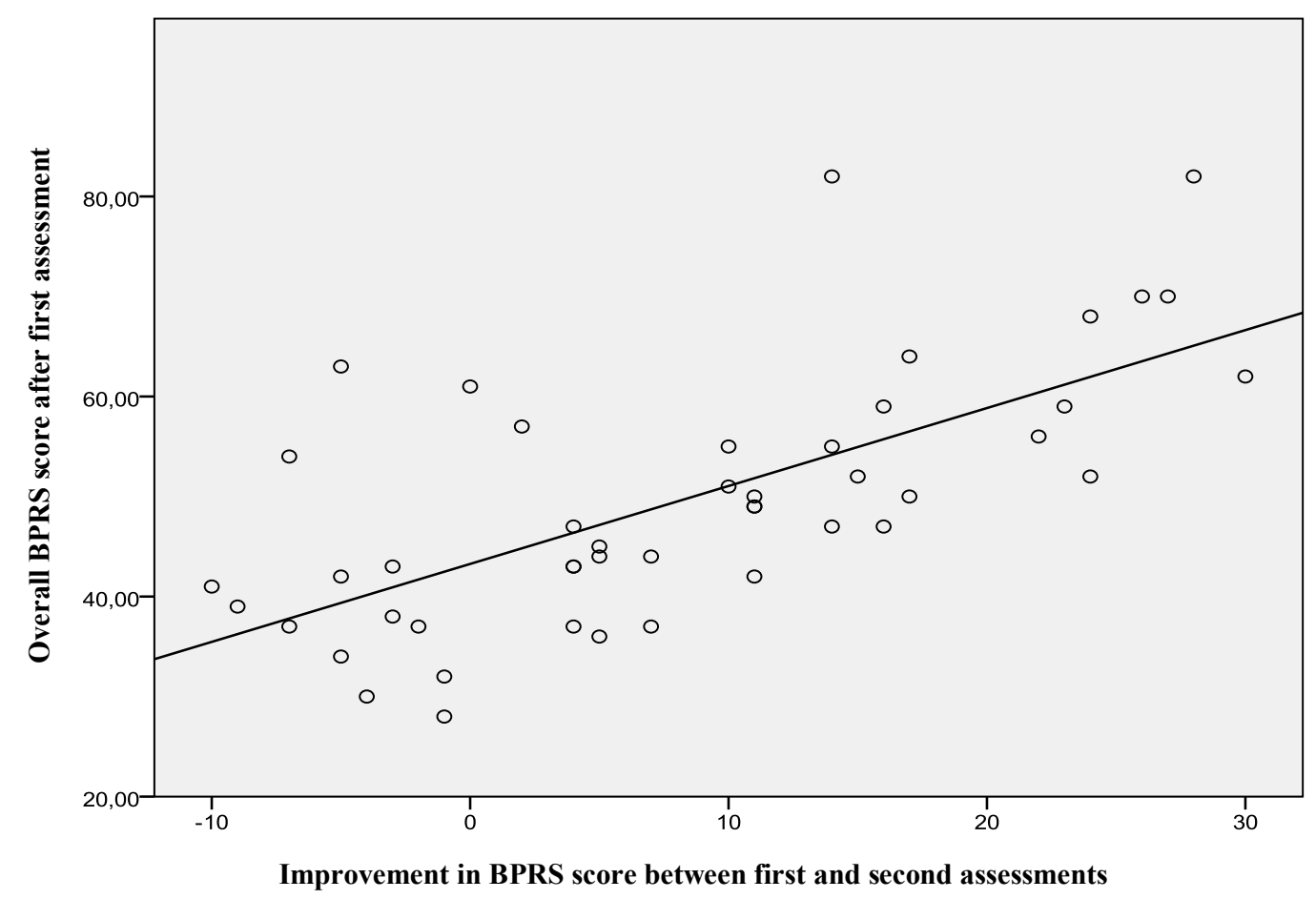

Graph 1: Correlation between initial score and improvement between t0 and t1.

Table 11: Statistic.

\begin{tabular}{|l|l|l|l|}
\hline \multicolumn{2}{|l|}{ Analysis of the correlations (group 1) } & $\begin{array}{l}\text { Improvement in BPRS score between the } \\
\text { first and second assessments (t0 - t1) }\end{array}$ & $\begin{array}{l}\text { Improvement in BPRS score between the } \\
\text { first and second assessments (t0 - t1) }\end{array}$ \\
\hline $\begin{array}{l}\text { Overall BPRS score first } \\
\text { assessments (t0) }\end{array}$ & $\begin{array}{l}\text { Pearson's } \\
\text { correlation }\end{array}$ & 0.675 & 0.675 \\
\hline $\begin{array}{l}\text { Overall BPRS score first } \\
\text { assessment (t0) }\end{array}$ & $\begin{array}{l}\text { Significance } \\
\text { (2-tailed) }\end{array}$ & 0.000 & 0.000 \\
\hline & $\mathbf{N}$ & $\mathbf{4 6}$ & $\mathbf{4 6}$ \\
\hline
\end{tabular}

Table 12: BPRS improvement in group 2.

\begin{tabular}{|l|l|l|l|}
\hline Clinical evolution of BPRS scores (group 2) & Range & t0-t1 & t1-t2 \\
\hline Deterioration & Score increased by at least 24\% & $16.7 \%$ & $16.7 \%$ \\
\hline Stable & Score within +/- 24\% of the initial & $8.3 \%$ & $33.3 \%$ \\
\hline Slight improvement & Score diminished by 24 to 44\% & $25 \%$ & $33.3 \%$ \\
\hline $\begin{array}{l}\text { Alto miglioramento } \\
\text { Marked Improvement }\end{array}$ & $\begin{array}{l}\text { Punteggio diminuito almeno del 44\% Score diminished by } \\
\text { atleast 44\% }\end{array}$ & $50 \%$ & $17.7 \%$ \\
\end{tabular}

The evolution of scores between the first (t0) and the second (t1) assessments exhibits a strong correlation $(r=$ $0.675, p \leqq 0.01$ ) between the initial position ( $t 0$ ) on the BPRS scale and the improvement achieved after the first cycle of therapy (t0 - t1) (Table 11 and Graph 1).

In group 2 (psychotic patients that had no variations in pharmacological and/or rehabilitative-behavioral therapy) the average of the scores achieved using BPRS diminished between the start of treatment (t0) and the first follow up (t1) from 53.08 to 43.41 (difference $=9.67, \mathrm{p} \leqq 0.01$ ) (Table 12) and between the first and second follow up (t1 and t2) from 43.41 to 39.83 (difference $=3.58, p \leqq 0.05$ ) (Table 12 ).
The clinical significance of the evolution of scores between the start of treatment and the first follow up exhibited a deterioration in $16.7 \%$ of cases, a substantial stability in $8.3 \%$, a slight improvement in $25 \%$, and a marked improvement in $50 \%$. Between the first and second follow up there was a deterioration in $16.7 \%$ of cases, substantial stability in $33.3 \%$, a slight improvement in $33.3 \%$, and a marked improvement in $17.7 \%$ (Table 13).

\section{Discussion}

Ion cyclotron resonance-like is a biophysical phenomenon, the clinical application of which appears to be very effective in 
Citation: Betti M, Picchi MPC, Saettoni M, et al. (2019) Ion Cyclotron Resonance: Results and Prospects for Psychiatry. J Psychiatry Treat Res $1(1): 16-24$

Table 13: Statistical analysis of group 2

\begin{tabular}{|l|l|l|l|l|l|}
\hline \multicolumn{2}{|l|}{ Statistics per paired samples (group 2) } & Average & N. & Difference & Significance (p) \\
\hline Pair 1 & Overall BPRS score first assessment (t0) & 53.0833 & 12 & & \\
\cline { 2 - 6 } & Overall BPRS score second assessment (t1) & 43.4167 & 12 & 9.67 & $\leqq 0.01$ \\
\hline \multirow{2}{*}{ Pair 2 } & Overall BPRS score second assessment (t1) & 43.4167 & 12 & & \\
\cline { 2 - 5 } & Overall BPRS score third assessment (t2) & 39.8333 & 12 & 3.58 & $\leqq 0.05$ \\
\hline
\end{tabular}

different medical sectors, but that still needs to be explored in all its clinical and epistemological potential. The numerous studies conducted to date on the therapeutic effects of extremely weak electromagnetic fields appear to confirm the effectiveness of this methodology in various medical fields. Its practicality, ease of application, excellent tolerability, low cost, absence of invasiveness, and rareness of side effects, make it a very interesting instrument for the healthcare world.

The aim of the study was to verify the improvement obtained with this kind of treatment in patients who didn't have evidence of improvements by pharmacologic treatment stabilized for at least 1 year. During the trial period drug therapy was not changed.

As regards the psychiatry sector, the results achieved in the present study, notwithstanding all the limitations deriving from low sample numbers, lack of homogeneity, and absence of a control group, offer significant results from both the statistical and strictly clinical perspectives.

The sample of 46 subjects heterogeneous diagnoses achieved a reduction in BPRS score from 49.63 to 41.45 ( $p<=$ 0.01 ) and a slight or marked clinical improvement in $58.7 \%$ of cases. The sample comprising 12 subjects diagnoses of psychosis achieved an overall reduction in BPRS score from 53.08 to 39.83 (representing a reduction of 9.67 points, with ( $p<=0.01$ ) after the first treatment cycle, and of 3.58 points, with ( $p<=0.05$ ) after the second cycle) with a slight or marked clinical improvement in $75 \%$ of cases after the first treatment cycle, and in $50 \%$ of cases after the second. These positive results encourage further more accurate studies requiring the recruitment of a larger set of clinical cases, applying a more selective protocol for the administration of therapy and selection of subjects. For this purpose the authors are currently elaborating the necessary procedures for a double blind study. No T1-T2 or statistic is presented because we didn't observed a significative reduction of BPRS score.

Another interesting observation that emerged from the analyses of the results regards the clear correlation $(r=$ $0.675, p<=0.01$ ) between the gravity of symptoms before therapy and the positive evolution after the same. This would appear to indicate a greater effectiveness of ICR-like in cases characterized by greater initial seriousness, which would confirm the authors' expectations regarding a therapeutic action affecting the biological substrate, which is more extremely compromised in the most serious clinical pictures.

An increase of the research on the applications of ICR-like would make it possible to formulate new intervention strategies. First of all, there are extensive opportunities for integration with other types of treatment psychopharmacological, psychotherapeutic, or rehabilitative - establishing therapeutic courses capable of acting at different levels of complexity. Furthermore, the promotion of enzymatic processes, reduction in oxidative stress, and stimulation of neurotrophic factors are all non-specific components that improve general health and wellbeing, regardless of the symptomatic picture.

This approach opens up numerous promising alternatives and possibilities: on one hand there is an action that could be defined as "non-specific", as the biophysical alterations occur in many forms of pathology and represent an effect that could be termed "generic". On the other hand, treatments based essentially on electromagnetic parameters act on a finer level than the more familiar chemical-pharmacological remedies. The possibility of inducing the ion resonance effect in a particular organism and in a given situation lays down the foundations for more closely targeted and customized treatments. Effectively, this represents a paradigm shift that calls for the definition of new assessment criteria and a radical reformulation of the old nosographic classifications.

Additional opportunities for development and research into ICR-like effects are presented by the accessory device used in the study and referred to as a "probe". This technical accessory makes it possible to direct an electromagnetic beam, focusing the effect on specific somatic zones. In this way a bridge can be created between biophysical and psychotherapeutic treatments, opening up new prospects for investigative treatment.

An integration of this methodology with the more recent developments in cognitive approaches is feasible, especially Mindfulness and Body Scanning, as well as with psychocorporeal therapies derived from post-Reichian ideas and bioenergetics, aiming at the release of neuromotor blocks and tensions.

\section{Conclusions}

In confirmation of the results of the previous study, treatment with IRC-like demonstrated a significant effectiveness in the psychiatric disorders examined, with a marked effect in the more serious cases. The expectations formulated on the basis of the mechanism of action hypotheses, appear to be further confirmed by these conclusions.

As research develops, the authors propose optimization of treatment protocols, times, and methods as well as identification of the most sensitive target pathologies. 


\section{Conflicts of Interest}

Dr. Alessandro Grecodeclares that he provides scientific consultation in the development of new medical research for the company that manufactures the electromagnetic device used.

The other authors declare that no conflicts of interest exist. All costs for the present study were covered by the Public Health Service of Regione Toscana (Italy).

\section{Acknowledgments}

We thank the USL Toscana Nord Ovest for their support. We also thank Professor R. Abraham Liboff for initiating studies on Ion Cyclotron Resonance.

\section{References}

1. Barker AT, Jalinous R, Freeston IL (1985) Non-invasive magnetic stimulation of human motor cortex. Lancet 1: 1106-1107.

2. George MS, Bellmaker RH (2000) Transcranial magnetic stimulation in neuro-psychiatry. American Psychiatric Association Press, Washington DC, USA.

3. Raggi F, Vallesi G (2010) The therapeutic use of ELF magnetic fields: The evidences in the literature. Italian Electrodynamic Biophysics Society, Proceedings of the III National Conference: From biological effects to interpretations, from case reports to clinical practice. Ultra-weak magnetic fields and Ciclotronica lonorisonanza, a concrete aid in regenerative medicine. Bologna, 29-34.

4. Liboff AR (1985) Geomagnetic cyclotron resonance in living cells. Journal of Biological Physics 13: 99-102.

5. Liboff AR, Jenrow KA (2002) Physical mechanisms in neuroelectromagnetic therapies. NeuroRehabilitation 17: 9-22.

6. Wright HR, Lack LC, Kennaway DJ (2004) Differential effects of light wavelength in phase advancing the melatonin rhythm. J Pineal Res 36: 140-144.

7. Terman M, Terman JS (2005) Light therapy for seasonal and nonseasonal depression: Efficacy, protocol, safety, and side effects. CNS Spectr 10: 647-663.

8. Even C, Schröder CM, Friedman S, Rouillon F (2008) Efficacy of light therapy in nonseasonal depression: A systematic review. J Affect Disord 108: 11-23.

9. Gooley JJ, Rajaratnam SMW, Brainard GC, et al. (2010) Spectral responses of the human circadian system depend on the irradiance and duration of exposure to light. Sci Transl Med 2: 31-33.

10. Markov MS (2007) Magnetic field therapt: A review. Electromagn Biol Med 26: 1-23.

11. Crescentini $F$ (2007) The autistic syndrome and endogenous ion cyclotron resonance: State of the art. Electromagn Biol Med 26: 305-309.

12. Sandyk R (1994) Improvement in word-fluency performance in Parkinson's disease by administration of electromagnetic fields. Int J Neurosci 77: 23-46.

13. Sandyk R (1994) Alzheimer's disease: Improvement of visual memory and visuoconstructive performance by tratment with picotesla range magnetic fields. Int J Neurosci 76: 185-225.

14. Sandyk R (1994) Improvement in word-fluency performance in patients with multiple sclerosis by electromagnetic fields. Int J Neurosci 79: 75-90.
15. Lappin MS, Lawrie FW, Richards TL, et al. (2003) Effects of a pulsed electromagnetic therapy on multiple sclerosis fatigue and quality of life: A double-blind, placebo controlled trial. Altern Ther Health Med 9: 38-48.

16. Rossi E (2010) Ipotesi sul meccanismo terapeuticodgli ELF EMF. Società Italiana Biofisica Elettrodinamica, Atti del III Convegno Nazionale: Dagli effetti biologici alle interpretazioni, dai case report alla prassi clinica. I campi magnetici ultradeboli e la Ionorisonanza Ciclotronica, un aiuto concreto in medicina rigenerativa. Bologna, 35-37.

17. Jenrow KA, Liboff AR (2003) Electromagnetic techniques in neural therapy. In: Rosch PJ, Markov M, Bioelectromagnetic Medicine. Dekker, New York.

18. Sánchez R, Alcoverro O, Pagerols J, et al. (2009) Electrophysiological mechanisms of action of electroconvulsive therapy. Actas Esp Psiquiatr 37: 343-351.

19. Duman R.S, Vaidya VA (1998) Molecular and cellular actions of chronic electroconvulsive seizures. J ECT 14: 181-193.

20. Hameroff SR, Watt RC (1982) Information processing in microtubules. Journal of Theoretical Biology 98: 549-561.

21. Sandyk R, Anninos PA, Tsagas N (1991) Magnetic fields and seasonality of affective illness: Implications for therapy. Int J Neurosci 58: 261-267.

22. Conca A, Koppi S, König P, et al. (1996) Transcranial magnetic stimulation: A novel antidepressive strategy? Neuropsychobiology 34: 204-207.

23. Dell'Osso B, Camuri G, Castellano F, et al. (2011) Meta-Review of metanalytic studies with repetitive transcranial magnetic stimulation (rTMS) for the treatment of major depression. Clin Pract Epidemiol Ment Health 7: 167-177.

24. Liboff AR (1994) The electromagnetic field as a biological variable. In: Frey $\mathrm{AH}$, On the nature of electromagnetic field interactions. R.G. Landis, Austin.

25. Liboff AR (2003) The charge-to-mass ICR signature in weak ELF bioelectromagnetic effects. In: Lin J, Advances electromagnetic fields in living systems. Kluwer/Plenum, New York.

26. Pazvantoglu O, Selek S, Okai T, et al. (2009) Oxidative mechanisms in schizophrenia and their relationship with illness subtype and symptom profile. Psychiatry Clin Neurosci 63: 693-700.

27. Betti M, Di Meo MC, Picchi MPC, et al. (2010) Applicazioni della Ionorisonanza Ciclotronica in salute mentale. Società Italiana Biofisica Elettrodinamica, Atti del III Convegno Nazionale: Dagli effetti biologici alle interpretazioni, dai case report alla prassi clinica. I campi magnetici ultradeboli e la lonorisonanza Ciclotronica, un aiuto concreto in medicina rigenerativa. Bologna 41-50.

28. Morosini P, Roncone R, Impallomeni Maria, et al. (1994) Presentazione dell'adattamento italiano della Brief Psychiatric Rating Scale, versione 4.0 ampliata (BPRS 4.0). Psychiatric and Psychosocial Rehabilitation Magazine 3: 195-198.

29. Ventura J, Green MF, A Shaner, et al. (1993) Training and quality assurance with the brief psychiatric rating scale: "The drift busters". International Journal of Methods in Psychiatric Research 3: 221-244.

30. Leucht S, Kane John M, Kissling W, et al. (2005) Clinical implications of brief psychiatric rating scale scores. Br J Psychiatry 187: 366-371. 